\title{
PROTOTIPADO RÁPIDO EN LA FABRICACIÓN DE MOLDES IMPRESOS EN 3D PARA SOPLADO DE PLÁSTICO
}

\section{RAPID PROTOTYPING IN THE MANUFACTURE OF 3D PRINTED MOLDS FOR PLASTIC BLOWING}

\author{
Gilberto Carrillo ${ }^{1, *}$, Carolina Nuila², Jorge Laínez ${ }^{3}$
}

\section{Resumen}

En la industria salvadoreña pueden encontrarse empresarios y microempresarios que no tienen los recursos para fabricar botellas de plástico con diseños estilizados que los diferencien de otras marcas y productos, lo que les impide escalar a otros segmentos de mercado o mercados internacionales, frenando el crecimiento de sus negocios. Una posible causa es que la fabricación de moldes de soplado requiere una inversión inicial muy costosa. Sin embargo, existen alternativas como la fabricación de moldes de bajo rendimiento, que tienen una resolución más baja y un tiempo de vida más corto, pero, al mismo tiempo, ofrecen como beneficio un menor costo de fabricación y, por lo tanto, un menor costo de adquisición para el empresario, posibilitando la producción de botellas estilizadas a conveniencia. Entre las diversas formas de fabricar moldes de bajo rendimiento está la técnica de ingeniería inversa, que requiere un equipo de creación rápida de prototipos. Este artículo describe el procedimiento de ingeniería inversa para generar el molde para soplado. Con el diseño disponible se imprimió el molde necesario y con esto se fabricaron las botellas, que se escanearon para verificar con el programa de computadora sus dimensiones comparándolas con el archivo original del molde. Simultáneamente, los contenedores se verificaron en el laboratorio de metrología industrial para validar los resultados de la computadora, estos resultados se presentan en el documento.

Palabras clave: molde para soplado, impresión 3D, creación rápida de prototipos, botellas de plástico

\section{Abstract}

In the Salvadoran industry, we can find entrepreneurs and microentrepreneurs who do not have the resources to make plastic bottles with stylized designs that differentiate them from other brands and products, which prevents them from escalating to other market segments or international markets, slows the growth of their business. One possible cause is that the manufacture of blow molds requires a very expensive initial investment. However, there are alternatives such as the manufacture of low-run molds, which have lower resolution and shorter life time, but, at the same time, offer as a benefit a lower manufacturing cost and, therefore, lower acquisition cost for the entrepreneur, opening in this way the opportunity to be able to produce stylized bottles at convenience. Among the various ways to manufacture low-run molds, there is the reverse engineering technique, which requires rapid prototyping equipment. This article describes the reverse engineering procedure to generate the mold for blowing. With the available design the necessary mold was printed and with this, the bottles were manufactured, which were scanned to verify with computer program their dimensions comparing them against the original mold file. Simultaneously, the containers were verified in the industrial metrology laboratory to validate the computer results, these results are presented in the document.

Keywords: Mold for blowing, 3D printing, rapid prototyping, plastic bottles.

\footnotetext{
$\overline{1, *}$ Centro de Innovación en Diseño Industrial y Manufactura, Universidad Don Bosco, El Salvador. Autor para correspondencia : gilberto.carrillo@udb.edu.sv. (1) http://orcid.org/0000-0002-9845-1381

${ }^{2}$ Laboratorio de Metrología, Universidad Don Bosco, El Salvador. (D) http://orcid.org/0000-0002-7626-389X

${ }^{3}$ Herramientas Centroamericanas S. A. de C. V., El Salvador. (D) http://orcid.org/0000-0001-7940-5366
}

Recibido: 04-02-2020, aprobado tras revisión: 06-05-2020

Forma sugerida de citación: Carrillo, G.; Nuila, C. y Laínez, J. (2020). «Prototipado rápido en la fabricación de moldes impresos en 3D para soplado de plástico». InGEnIUS. N. ${ }^{\circ}$ 24, (julio-diciembre). pp. 28-35. DOI: https: //doi.org/10.17163/ings.n24.2020.03. 


\section{Introducción}

Los fabricantes de recipientes de plástico y los empresarios salvadoreños no pueden adquirir nuevos moldes de aleación de aluminio y acero para nuevos productos, ya que esto requiere una alta inversión inicial que solo puede ser amortizada con una elevada producción de productos [1]. Como ejemplo, las versiones metálicas del molde de soplado de botellas pueden costar desde 2000 hasta 5000 USD, los cuales si se fabrican regionalmente pueden recibirse entre 30 y 60 días, y fabricados en Europa o Norteamérica puede recibirse desde 90 hasta 150 días [2]. Esto implica que las micro, pequeñas y medianas compañías salvadoreñas, con bajos márgenes de ganancia, no pueden incorporar en su oferta nuevos productos con recipientes de plásticos diferenciados.

Como una solución alternativa a los moldes, la impresión 3D se convierte en una «innovación tecnológica revolucionaria impulsada por la flexibilidad que proporciona y la economía potencialmente favorable» [3]. Otras aplicaciones de los moldes impresos son su uso en la fabricación a mano de piezas compuestas [4], fabricación de piezas plásticas [5], desarrollo de moldes de inyección de cera de bajo costo [6]. Mientras que altera los enfoques tradicionales de fabricación y promueve la expansión de tecnologías de prototipado rápido y fabricación digital.

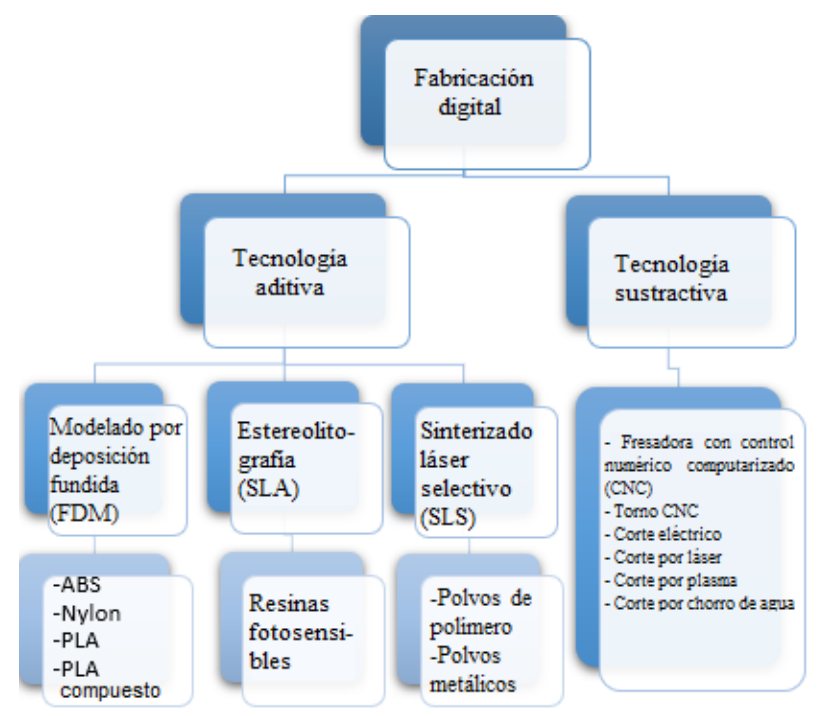

Figura 1. Técnicas de fabricación digital

Como se muestra en la Figura 1, las tecnologías de fabricación digital pueden clasificarse en aditivas, como impresión 3D, y sustractivas, como equipos de corte con CNC (control numérico computarizado).

Existen prácticas en los Estados Unidos y Europa, donde utilizan tecnologías aditivas tales como SLA (Estereolitografía, Stereolithography por sus siglas en inglés) y SLS (Sinterizado Láser Selectivo) en la fabricación de moldes por inyección [7]. Estas tecnologías son de elevada inversión y útiles en la producción a pequeña escala.

Hasta donde saben los autores, la técnica de FDM (modelado por deposición fundida, Fused Deposition Modeling por sus siglas en inglés) ha sido poco utilizada en la fabricación de moldes. Este trabajo presenta la fabricación de un molde por medio de la tecnología FDM de prototipado rápido y baja inversión, con la cual se producen recipientes con diseños estilizados para cada necesidad.

\section{Materiales y métodos}

Considerando las técnicas de modelado, los métodos de generación de moldes podrían clasificarse en ingeniería inversa y proceso CAD (Computer Aided Design, diseño asistido por computadora por sus siglas en inglés), representado en la Figura 2 y descrito en 5 etapas.

Aplicando ingeniería inversa, se escaneó un recipiente suministrado por la industria, a partir del cual se obtuvo el molde para ser estudiado.

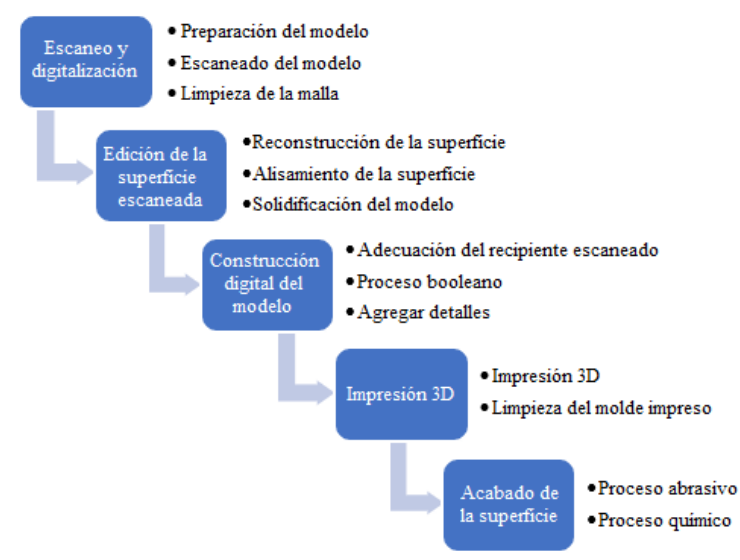

Figura 2. Etapas de aplicación del prototipado rápido

En la etapa 1, se utilizó el escaneado y el equipo de escaneado SmartScan R2-C2 con una precisión de 0,01 mm y su software Optocat 2015®. El líquido revelador es CANTESCO D101-A para atenuar el brillo y la reflexión de las superficies a ser escaneadas. El procedimiento de escaneado se hace de conformidad con el manual de usuario OPTOCAT versión 2015R2 [8].

La temperatura del salón de prototipado rápido se mantuvo en condiciones estables $\left(24{ }^{\circ} \mathrm{C}\right)$, y la entrada de luz desde el exterior se redujo menos de $25 \%$ de la imagen en directo iluminada para evitar el ruido en las imágenes escaneadas debido a las variaciones de la luz. La nube final de puntos es almacenada en un archivo con formato STL (lenguaje estándar de triángulo, Standard Triangle Language), para luego trabajar en otro software.

En la etapa 2 se realizó la edición de la superficie escaneada con el tratamiento de la nube de puntos, 
utilizando el software CAD GeoMagic Design X. El archivo generado fue almacenado en formato STEP (estándar para el intercambio de información de producto, Standard for the Exchange of Product Data), que evita la pérdida de información de diseño.

Para la etapa 3 se llevó a cabo la construcción del molde con el software Inventor. Se incorporaron las dimensiones del bloque, los agujeros de alineación, la rosca, los agujeros de escape de aire y el diseño de la boquilla.

Dentro de la etapa 4, el material utilizado en la impresión 3D de los moldes de soplado fue el ABS-plus (acrilonitrilo butadieno estireno) P430-XL, el material de soporte es SR-30XL (soporte soluble), el material de las botellas es PET (polietileno tereftalato). Asimismo, el proceso de impresión 3D fue realizado de acuerdo con el manual de usuario de la impresora uPrint SE Plus; el software utilizado se llama CatalystEX y el sistema de eliminación de soporte es el equipo WaveWash que trabaja por medio de lavado ultrasónico [9]. El molde fue utilizado en la compañía POLIFLEX para fabricar 25 botellas de prueba. Con esta primera corrida surgen las recomendaciones que constituyen la etapa 5 .

En la misma, el acabado de la superficie del molde fue hecho con masilla automotriz en la cavidad donde se forma el recipiente. El lijado manual fue realizado con papel de lija 1000 para mejorar la suavidad de la superficie, y facilitar el deslizamiento del PET durante el soplado.

Fuera del proceso de ingeniería inversa, los recipientes fueron escaneados para ser comparados por medio de software, y se realizaron pruebas en el laboratorio de metrología industrial para validar sus resultados.

\section{Resultados y discusión}

En esta sección se presentan los resultados en el orden de los bloques mostrado en la Figura 2.

Etapa 1. Una nube de puntos fue obtenida del proceso de escaneado y digitalización, tal como se muestra en la Figura 3. No fue necesario escanear la boquilla del recipiente, debido a que el diseño de dicha boquilla fue incorporado en la etapa posterior de construcción digital, de acuerdo con la máquina sopladora. La posibilidad de cambiar de boquilla en el molde ocurre cuando se planea producir recipientes con máquinas que requieren preformas.

Etapa 2. La edición de la superficie escaneada consiste de un conjunto de operaciones para obtener un cuerpo alisado, como corte, combinación, reparación, alisado de superficies y bordes. El archivo STL puede mejorarse con cualquier software CAD.

La botella final se muestra en la Figura 4, y puede incorporar detalles finales característicos tales como formas adicionales, áreas para etiquetado, textos y logos en alto y bajorrelieve, etc.

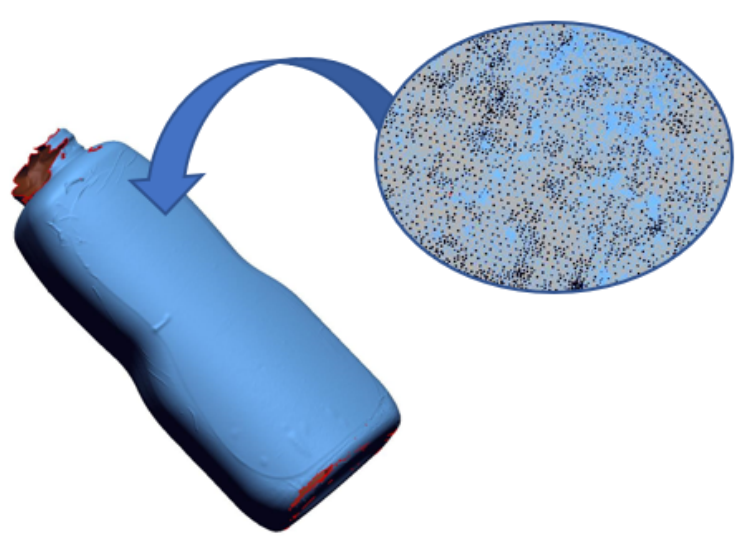

Figura 3. Nube de puntos del recipiente con formato STL, resultante del proceso de escaneado 3D y de escaneado y listo para ser procesado con software CAD
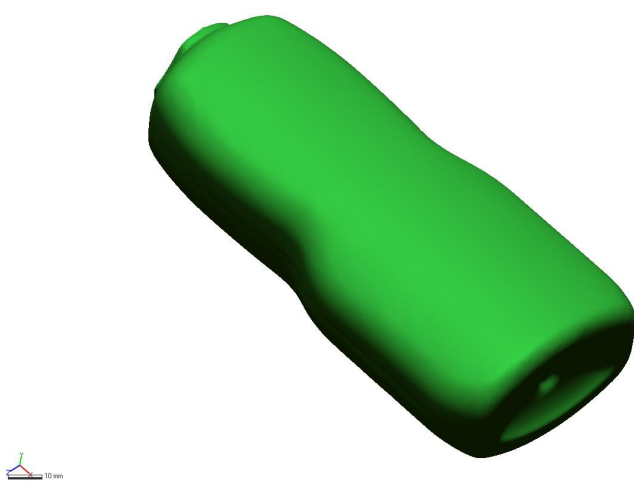

Figura 4. Botella alisada por computadora y acabada

Etapa 3. La construcción digital del molde consiste en la transformación de la botella de la etapa previa (etapa 2) a sólido en el CAD. El cuerpo sólido en forma de media botella es adherido por medio de un proceso de corte de objetos sólidos en el software CAD, y posteriormente se agregan los detalles del molde, como por ejemplo, los agujeros de fijación, roscas, agujeros de escape y el espacio de la boquilla.

Algunas consideraciones importantes previstas en la digitalización del molde, y que tienen influencia sobre la calidad de los productos son:

- No existieron defectos en la superposición de los volúmenes del recipiente y del molde.

- El espesor de las superficies en los bordes permitió resistir las cargas recibidas.

- Las curvaturas de las geometrías y de las superficies son correctas para reducir la concentración de esfuerzos. 
- Se indicó la alineación de agujeros, pernos, superficies y bordes para evitar irregularidades en las superficies de productos manufacturados [10].

- Las tolerancias de los agujeros fueron correctas para que ambas partes pudieran ser acopladas durante la producción de recipientes con ajuste deslizante [11].

- Las dimensiones del escape de aire fueron adecuadas para que no se vieran marcas en los recipientes manufacturados.

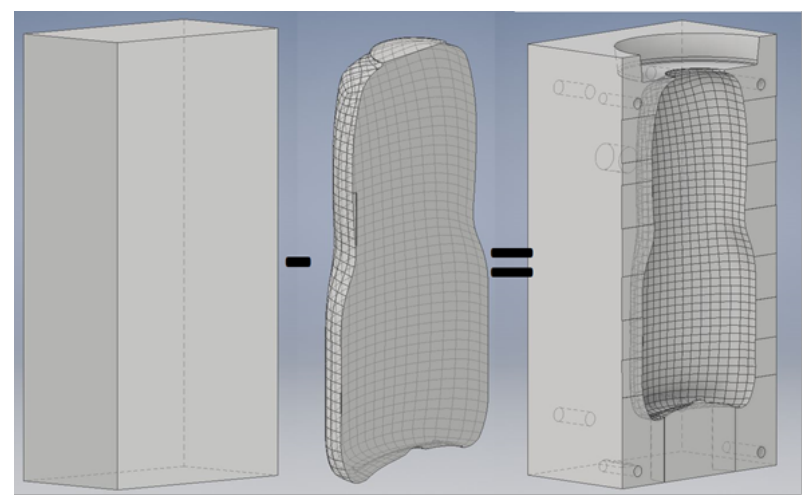

Figura 5. Secuencia de procesos para la fabricación de un molde: El cuerpo sólido en forma de media botella es adherido por medio de un proceso de corte de objetos sólidos en el software CAD, y posteriormente se agregan los detalles del molde, como por ejemplo, los agujeros de fijación, respiraderos y el espacio de la boquilla

La boquilla depende del tipo de máquina donde será instalado el molde y el tipo de materia prima. Por ejemplo, la boquilla de la máquina de moldeo por soplado forma la rosca externa y utiliza material granulado, mientras que la boquilla de la máquina de preformas es lisa porque esta recibe las preformas con su rosca ya fabricada. Con esta información se dibujó una boquilla al molde, como se muestra en la Figura 5.

Los moldes fueron diseñados con un fondo removible, el cual debe ser removido durante el desmoldado del recipiente, e instalado durante el cierre del molde. $\mathrm{Su}$ función es evacuar el aire durante el soplado, y formar el fondo del recipiente para proporcionarle estabilidad mientras permanece en superficies horizontales planas.

El molde diseñado fue analizado por medio de una computadora con el software CAE Inventor (ingeniería asistida por computadora, Computer Aided Engineering), aplicando una presión de 350 psi en las superficies curvas, la cual es la presión de soplado para la fabricación de botellas. Los resultados se muestran en la Figura 6 , donde se observa una deformación promedio de 0,66 $\mathrm{mm}$ representada por el área verde, y una deformación máxima de 1,026 mm en el fondo del molde representada por el área roja.

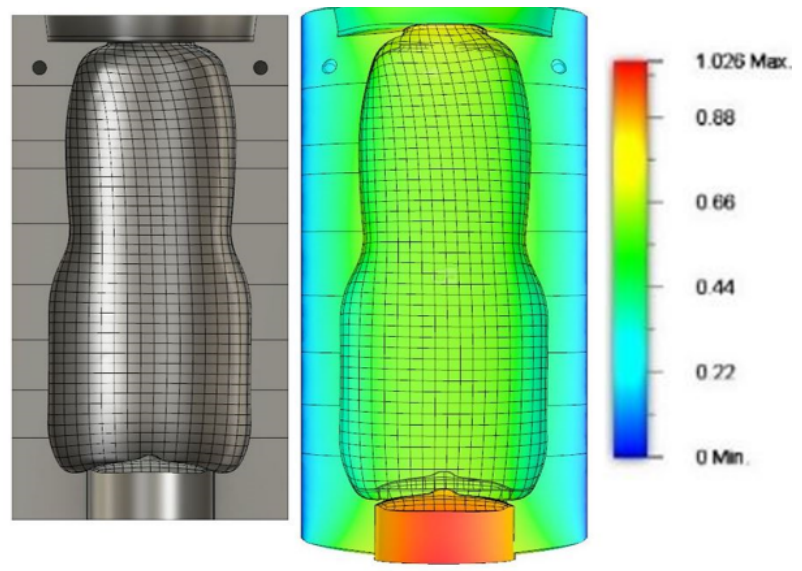

Figura 6. Puntos críticos de la deformación del molde, considerando los puntos de concentración de esfuerzos calculados por el software y mostrados en la paleta de colores. La presión de carga simulada fue 350 psi, la temperatura de análisis $100{ }^{\circ} \mathrm{C}$, y las unidades de la deformación se muestran en la escala de colores en milímetros

Etapa 4. ABS fue utilizado como material de impresión, Figura 7. La altura de las capas de impresión es $0,25 \mathrm{~mm}$, el espesor de la carcasa de la superficie exterior $10 \mathrm{~mm}$, la densidad de relleno en la pared $100 \%$, la densidad de relleno en el medio $60 \%$, el espesor de las capas sólidas tope/fondo $10 \mathrm{~mm}$, con el equipo uPrint SE plus que genera superficies curvas con baja resolución. Esto es una dificultad cuando se necesitan botellas lisas, mientras que es una ventaja para diseños estilizados con superficie irregular. En este caso el fondo del molde fue impreso como parte de una de las mitades y aunque mostró cierta interferencia en el desmoldado, afectó 2 botellas de 25, por lo que se consideró que no tuvo efecto negativo durante la fabricación.

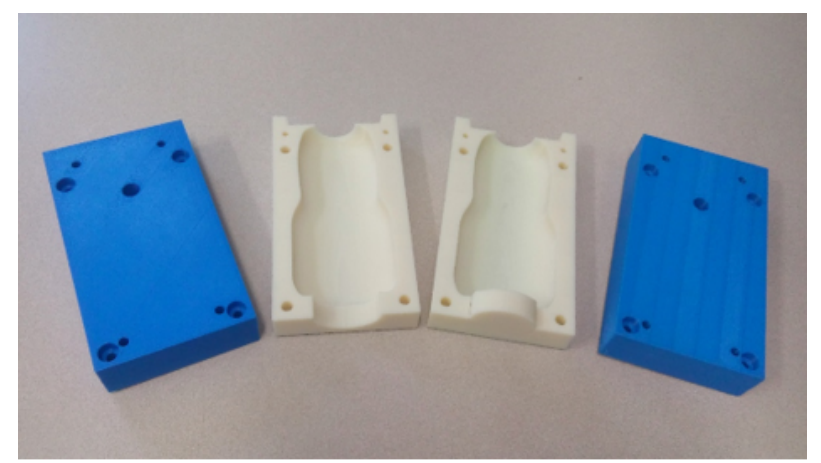

Figura 7. Moldes de soplado para botellas impresas en ABS

El molde para soplado de botellas se muestra en la Figura 7. El molde para impresión 3D incluye material ABS y material de soporte suministrados por 
proveedores en El Salvador. La Tabla 1 muestra los costos de impresión 3D. El costo principal corresponde a los materiales y al tiempo del diseñador; el costo de los materiales puede reducirse utilizando una impresora 3D de código abierto, y permite investigar acerca de nuevos materiales para impresión 3D con mejores propiedades mecánicas. El tiempo de trabajo en CAD puede optimizarse practicando para mejorar las habilidades.

Tabla 1. Costo de los moldes 3D impresos, utilizando precios de materiales existentes en el mercado salvadoreño

\begin{tabular}{|c|c|c|}
\hline \multicolumn{3}{|c|}{ Costo del molde de impresión 3D } \\
\hline Material de impresión (centímetros cúbicos) & 552.48 & \\
\hline Material de soporte (centímetros cúbicos) & 227 & \\
\hline Material de impresión, $\mathrm{cm}^{3}$ (costo unitario) & $\$ 0.42$ & \\
\hline Material de soporte, $\mathrm{cm}^{3}$ (costo unitario) & $\$ 0.42$ & \\
\hline Costo total del material de impresión & $\$ 232.04$ & $\$ 232.04$ \\
\hline Costo total del material de soporte & $\$ 95.34$ & $\$ 95.34$ \\
\hline Costo de la energía para impresión & $\$ 0.86$ & \\
\hline Amortización por hora & $\$ 2.65$ & \\
\hline Tiempo de impresión & 12 & \\
\hline Potencia $(\mathrm{KW})$ & 1.5 & \\
\hline Amortización durante la impresión & $\$ 3.98$ & \\
\hline IVA (El Salvador) & $13 \%$ & \\
\hline Costo total de la energía & $\$ 21.98$ & $\$ 21.98$ \\
\hline Diseño/configuración (horas) & 14 & \\
\hline Diseño/configuración (costo unitario) & $\$ 10.00$ & \\
\hline Costo total diseño/configuración & $\$ 140.00$ & $\$ 140.00$ \\
\hline Costo total del molde de impresión 3D & & $\$ 489.36$ \\
\hline
\end{tabular}

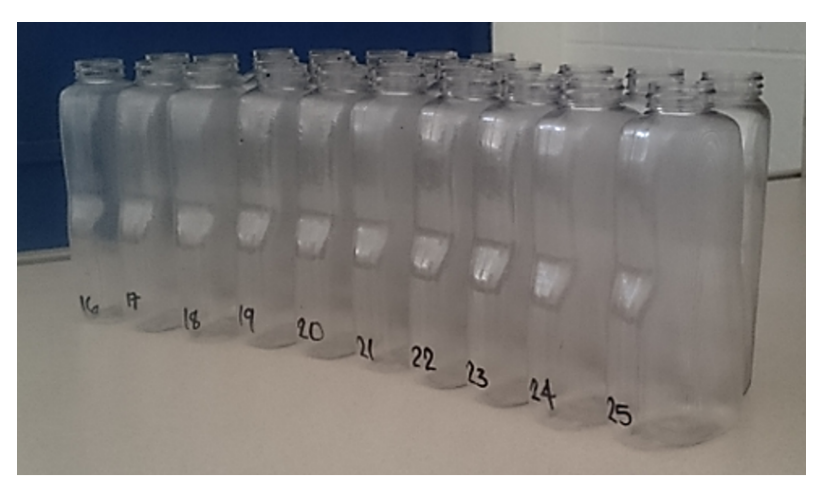

Figura 8. Botellas plásticas hechas con el uso de moldes impresos

Utilizando el molde impreso, se obtuvieron 25 recipientes en una compañía de producción; el molde fue instalado en una de sus máquinas sopladoras y se produjeron botellas, como se muestra en la Figura 8. El molde y las botellas fueron verificados con la ayuda de un escáner, determinándose que estaban en buenas condiciones dimensionales. Se considera que las superficies de interés para ser evaluadas son aquellas que soportaron la presión de las preformas durante la operación de soplado, y las superficies que soportaron la presión de cierre de las máquinas.

Las botellas fueron escaneadas y comparadas con el diseño del molde en el formato CAD. La Figura 9 muestra los puntos de los recipientes verificados digitalmente, según los puntos críticos resultantes del análisis del molde en la Figura 6 con el CAE Inventor. En términos generales, las dimensiones de los recipientes están en el rango de un décimo y medio milímetro por encima de las dimensiones promedio, y tres décimos de milímetro por debajo del promedio.

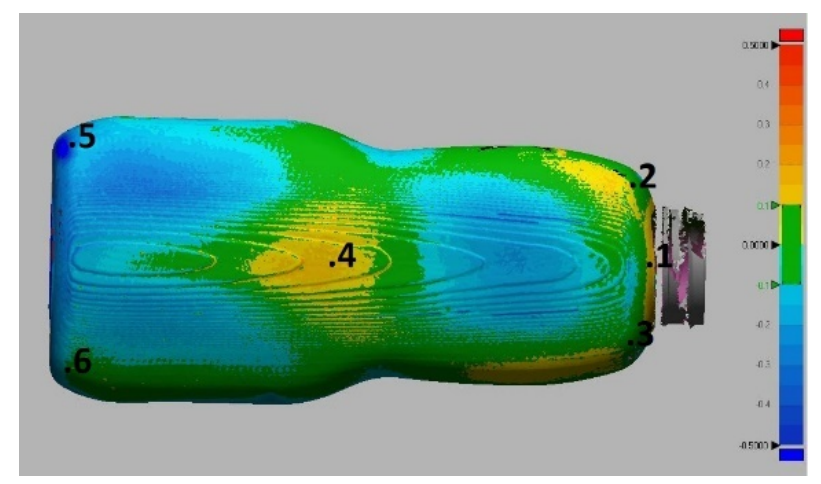

Figura 9. Verificación digital de las dimensiones de los recipientes producidos con el molde impreso junto con su paleta de colores

Los colores azul, azul claro, verde y amarillo en la Figura 9 denotan desviaciones mínimas del modelo escaneado con respecto al modelo digital, por lo que se verifica que las botellas fabricadas se mantienen en los rangos de $+0,15$ y $-0,2 \mathrm{~mm}$. Los resultados de la medición obtenidos con la técnica digital se muestran en la Tabla 2. El color marrón denota una alta desviación positiva/negativa y el color amarillo indica baja desviación positiva/negativa, a partir de las dimensiones promedio; las dimensiones son $\mathrm{A}=168,168 \mathrm{~mm}$ (alto), $\mathrm{B}=61,130 \mathrm{~mm}$ (anchura superior), $\mathrm{C}=72,610 \mathrm{~mm}$ (anchura inferior), $\mathrm{D}=41,518 \mathrm{~mm}$ (profundidad superior), $\mathrm{E}=43,049$ (profundidad inferior).

Los recipientes fueron analizados en el laboratorio de metrología para verificar hermeticidad, dimensiones y peso [12]. Los puntos de verificación y parte del equipo utilizado se muestran en la Figura 10, y la Tabla 3 da a conocer un extracto de los valores promedio y extremos determinados. De manera similar, la Tabla 4 presenta un extracto de los pesos determinados, indicando los valores extremos y promedio. Es importante notar que ninguna de las botellas presenta fugas luego de ser probadas con agua. 
Tabla 2. Resultados de la diferencia en las mediciones entre los recipientes producidos y el molde impreso

\begin{tabular}{ccccccc}
\hline $\begin{array}{l}\text { Numero } \\
\text { botella }\end{array}$ & Punto 1 & Punto 2 & Punto 3 & Punto 4 & Punto 5 & Punto 6 \\
\hline $\mathbf{1}$ & 0.0723 & 0.1644 & 0.0917 & 0.0881 & -0.1216 & 0.0280 \\
$\mathbf{2}$ & 0.1439 & 0.1445 & 0.0832 & 0.0277 & -0.0110 & 0.0636 \\
$\mathbf{3}$ & 0.1182 & 0.1125 & 0.0790 & 0.0624 & -0.0580 & 0.0896 \\
$\mathbf{4}$ & 0.0175 & 0.1001 & 0.1109 & 0.0551 & -0.0569 & 0.3939 \\
$\mathbf{5}$ & 0.0784 & 0.0383 & 0.0622 & 0.1062 & -0.1231 & 0.0720 \\
$\mathbf{6}$ & 0.1094 & 0.0512 & 0.0196 & 0.1632 & -0.2330 & -0.0138 \\
$\mathbf{7}$ & 0.0704 & 0.1229 & 0.0094 & 0.1078 & -0.2044 & 0.0223 \\
$\mathbf{8}$ & 0.0869 & 0.1268 & 0.0843 & 0.1458 & -0.2595 & -0.1917 \\
$\mathbf{9}$ & 0.1063 & 0.0729 & 0.0508 & 0.1260 & -0.1681 & -0.0795 \\
$\mathbf{1 0}$ & 0.1126 & 0.0972 & 0.0793 & 0.1312 & -0.3859 & -0.2118 \\
$\mathbf{1 1}$ & 0.1419 & 0.1103 & 0.1031 & 0.1056 & -0.2024 & -0.0397 \\
$\mathbf{1 2}$ & 0.1232 & 0.1455 & 0.1077 & 0.0906 & -0.3409 & -0.1720 \\
$\mathbf{1 3}$ & 0.1664 & 0.1377 & 0.1204 & 0.1109 & -0.1183 & -0.0979 \\
$\mathbf{1 4}$ & 0.1471 & 0.1288 & 0.0862 & 0.0909 & -0.2086 & -0.2159 \\
$\mathbf{1 5}$ & 0.1109 & 0.1257 & 0.0665 & 0.1428 & -0.1994 & -0.0293 \\
$\mathbf{1 6}$ & 0.1072 & 0.0673 & 0.0558 & 0.1033 & -0.3444 & -0.1224 \\
$\mathbf{1 7}$ & 0.1411 & 0.0258 & 0.0120 & 0.0973 & -0.3811 & -0.1013 \\
$\mathbf{1 8}$ & 0.1465 & 0.0938 & -0.0205 & 0.1325 & -0.3631 & -0.0562 \\
$\mathbf{1 9}$ & 0.1019 & 0.0603 & -0.0090 & 0.1041 & -0.3061 & -0.0247 \\
$\mathbf{2 0}$ & 0.1286 & 0.0966 & 0.0455 & 0.1397 & -0.1682 & -0.0201 \\
$\mathbf{2 1}$ & 0.1182 & 0.1136 & -0.0018 & 0.1095 & -0.2294 & -0.0770 \\
$\mathbf{2 2}$ & 0.0677 & 0.1241 & 0.0256 & 0.1068 & -0.1644 & -0.0080 \\
$\mathbf{2 3}$ & 0.1159 & 0.1199 & 0.0478 & 0.1164 & -0.1837 & -0.0226 \\
$\mathbf{2 4}$ & 0.1147 & 0.1887 & 0.0476 & 0.1233 & -0.1654 & -0.0870 \\
$\mathbf{2 5}$ & 0.0905 & 0.1295 & 0.0803 & 0.0793 & -0.2773 & -0.0214 \\
\hline El equipo de verificación utilizó escáner 3D y el $50 f t w a r e$ GeoMagic. & \\
\hline & & & & & &
\end{tabular}

El equipo de verificación utilizó escáner 3D y el software GeoMagic.

Tabla 3. Diagrama de referencia para el análisis dimensional y ejecución de los test dimensionales con las dimensiones A alto, B anchura superior, C anchura inferior, D profundidad superior, E profundidad inferior

\begin{tabular}{|c|c|c|c|c|c|c|c|c|c|c|}
\hline \multicolumn{11}{|c|}{ Resultados de la verificación de las 25 botellas (mm) } \\
\hline Dimensión & \multicolumn{2}{|c|}{ A } & \multicolumn{2}{|c|}{ B } & \multicolumn{2}{|c|}{$\mathrm{C}$} & \multicolumn{2}{|c|}{ D } & \multicolumn{2}{|c|}{$\mathbf{E}$} \\
\hline Vref & \multicolumn{2}{|c|}{168.168} & \multicolumn{2}{|c|}{61.130} & \multicolumn{2}{|c|}{72.610} & \multicolumn{2}{|c|}{41.518} & \multicolumn{2}{|c|}{43.049} \\
\hline $\begin{array}{c}\text { ID de la } \\
\text { botella }\end{array}$ & $\begin{array}{c}\text { Valor } \\
\text { promedio } \\
\text { Vm }\end{array}$ & $\begin{array}{c}\text { Error } \\
\text { Vm - Vref }\end{array}$ & $\begin{array}{c}\text { Valor } \\
\text { promedio } \\
\text { Vm }\end{array}$ & $\begin{array}{c}\text { Error } \\
\text { Vm - Vref }\end{array}$ & $\begin{array}{c}\text { Valor } \\
\text { promedio } \\
\text { Vm }\end{array}$ & $\begin{array}{c}\text { Error } \\
\text { Vm - Vref }\end{array}$ & $\begin{array}{c}\text { Valor } \\
\text { promedio } \\
\text { Vm }\end{array}$ & $\begin{array}{c}\text { Error } \\
\text { Vm - Vref }\end{array}$ & $\begin{array}{c}\text { Valor } \\
\text { promedio } \\
\text { Vm }\end{array}$ & $\begin{array}{c}\text { Error } \\
\text { Vm - Vref }\end{array}$ \\
\hline 1 & 168.160 & 0.008 & 61.073 & 0.057 & 73.038 & -0.027 & 41.335 & 0.182 & 42.941 & 0.107 \\
\hline 2 & 168.219 & -0.051 & 61.075 & 0.056 & 73.003 & 0.007 & 41.250 & 0.267 & 42.907 & 0.142 \\
\hline 3 & 168.148 & 0.020 & 61.098 & 0.032 & 72.986 & 0.024 & 41.167 & 0.351 & 42.979 & 0.069 \\
\hline 4 & 168.173 & -0.005 & 61.089 & 0.041 & 72.981 & 0.029 & 41.335 & 0.183 & 42.980 & 0.069 \\
\hline 5 & 168.268 & -0.100 & 61.082 & 0.049 & 72.993 & 0.017 & 41.315 & 0.203 & 42.978 & 0.070 \\
\hline 12 & 168.162 & 0.006 & 61.137 & -0.007 & 73.000 & 0.011 & 41.758 & -0.240 & 42.990 & 0.059 \\
\hline 15 & 168.148 & 0.020 & 61.131 & -0.001 & 73.041 & -0.030 & 41.527 & -0.009 & 43.151 & -0.103 \\
\hline 18 & 168.139 & 0.029 & 61.173 & -0.042 & 73.051 & -0.041 & 41.604 & -0.087 & 43.109 & -0.060 \\
\hline 24 & 168.044 & 0.124 & 61.182 & -0.052 & 72.986 & 0.024 & 41.669 & -0.152 & 43.120 & -0.072 \\
\hline
\end{tabular}

Tabla 4. Resultados de la medición del peso de los 25 recipientes (mm)

\begin{tabular}{|c|c|c|}
\hline \multicolumn{3}{|c|}{ Resultados de la verificación de las 25 botellas (g) } \\
\hline \multicolumn{2}{|c|}{ Wref } & 283.538 \\
\hline ID de la botella & $\begin{array}{c}\text { Valor promedio } \\
\text { Wm }\end{array}$ & $\begin{array}{c}\text { Error } \\
\text { Wm - Wref }\end{array}$ \\
\hline 1 & 283.194 & -0.0345 \\
\hline 2 & 286.626 & 0.3087 \\
\hline 5 & 283.559 & 0.0021 \\
\hline 7 & 283.558 & 0.0020 \\
\hline 8 & 283.555 & 0.0016 \\
\hline 9 & 283.557 & 0.0018 \\
\hline 22 & 283.289 & -0.0249 \\
\hline 23 & 283.252 & -0.0287 \\
\hline 24 & 283.236 & -0.0302 \\
\hline 25 & 283.202 & -0.0336 \\
\hline
\end{tabular}



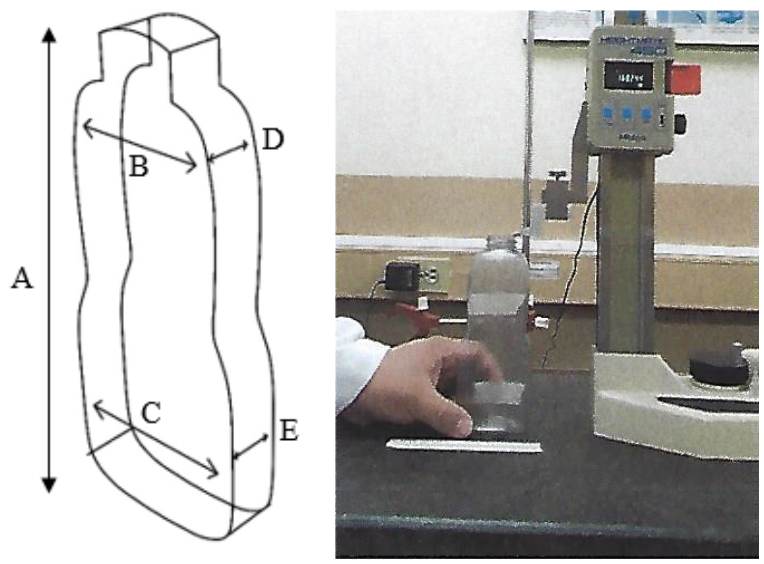

Figura 10. Diagrama de referencia para el análisis dimensional y ejecución de los test dimensionales con las dimensiones A alto, B anchura superior, C anchura inferior, D profundidad superior, E profundidad inferior

Etapa 5. Para mejorar el acabado de la superficie, se aplicó masilla automotriz al molde y se alisó con papel de lija de grado 1000, lo que resultó en una superficie lisa con rugosidad no evidente al toque. Esta operación es una variante del procedimiento normal de prototipado, donde los modelos impresos en FDM $3 \mathrm{D}$ son utilizados sin procesamiento ulterior. Este proceso artesanal es recomendado porque es barato y no toma mucho tiempo o hace al molde más costoso, lo cual se transforma en un beneficio para una SME de bajo presupuesto. La nota técnica de Hernández [13] se refiere al nivel de rugosidad o a los efectos de la suavidad en el flujo del parisón durante el soplado debido a la fricción contra la superficie del molde. Este hecho puede parecer contraproducente, sin embargo, luego de observar los primeros recipientes, la textura de la superficie puede ser un elemento distintivo para SME que pueden incorporar diferentes diseños en su empaque.

Un resultado útil para usuarios SME del molde es el tiempo que transcurre desde el escaneado hasta la entrega del molde acabado y limpio, que fue de 6 días. El tiempo de entrega es un factor positivo cuando se compara a los tiempos de entrega del molde a nivel regional y fuera de la región de Centroamérica.

\section{Conclusiones}

El trabajo realizado muestra que es posible aplicar ingeniería inversa para fabricar un molde impreso 3D con la técnica FDM [14]. Los recursos de la ingeniería inversa son usualmente equipo de escaneo 3D, computadoras y software de escaneo, equipo de impresión $3 \mathrm{D}$, y equipo de medición y verificación, entre otros.

El proceso de digitalización es replicable por instituciones que tienen un escáner 3D de alta definición, donde su producto será el archivo digital del molde. El archivo resultante será utilizado para fabricar el molde en cualquier institución que tenga una impresora 3D, cuando los recursos son escasos; sin embargo, cuando los recursos necesarios están disponibles, el archivo puede usarse para generar códigos que son introducidos en el equipo de fabricación de control numérico computarizado.

Este trabajo da una perspectiva para desarrollar investigación en tratamientos superficiales de moldes de polímero para el aumento de la durabilidad, estudio del uso de moldes de baja cadencia en procesos de termoformación, moldeado de plásticos biodegradables con el uso de moldes impresos y moldes para materiales compuestos reforzados. Estos resultados son antecedentes para aquellos que tienen software e impresoras 3D que pueden incorporarse en el campo productivo, ofreciendo servicios de diseño y fabricación de moldes impresos.

\section{Agradecimientos}

Esta investigación fue llevada a cabo gracias al apoyo financiero de la Agencia de los Estados Unidos para el Desarrollo Internacional (USAID, United States Agency for International Development), en su programa de Educación Superior para el Crecimiento Económico, el apoyo económico y la infraestructura de la Universidad Don Bosco, y la colaboración de la compañía Polietileno y Flexografía S. A. de C. V.

\section{Referencias}

[1] Ministerio de Economía de El Salvador, "Política nacional de fomento diversificación y transformación productiva," 2015. [Online]. Available: https://bit.ly/3fLvtCe

[2] R. Martell, Esperiencias en la empresa Roma Chemical, 2019.

[3] G. Unruh, "Circular economy, 3d printing, and the biosphere rules," California Management Review, vol. 60, no. 3, pp. 95-111, 2018. [Online]. Available: https://doi.org/10.1177/0008125618759684

[4] T. Z. Sudbury, R. Springfield, V. Kunc, and C. Duty, "An assessment of additive manufactured molds for hand-laid fiber reinforced composites," The International Journal of Advanced Manufacturing Technology, vol. 90, no. 5, pp. 1659-1664, May 2017. [Online]. Available: https://doi.org/10.1007/s00170-016-9464-9

[5] A. Baranov, A. I. Pronin, V. A. Dikov, A. V. Zakharov, and M. G. Lagutkin, "Casting molds for the routine production of plastic hydrocyclone components," Chemical and Petroleum Engineering, vol. 45, no. 7, p. 513, Oct. 2009. [Online]. Available: https://doi.org/10.1007/s10556-009-9219-7 
[6] C.-C. Kuo, W.-H. Chen, X.-Z. Liu, Y.-L. Liao, W.-J. Chen, B.-Y. Huang, and R.-L. Tsai, "Development of a low-cost wax injection mold with high cooling efficiency," The International Journal of Advanced Manufacturing Technology, vol. 93, no. 5, pp. 2081-2088, Nov. 2017. [Online]. Available: https://doi.org/10.1007/s00170-017-0596-3

[7] Sander Kunststofftechnik. (2017) Development and production of prototypes and small series made of plastic. [Online]. Available: https://bit.ly/3dAip0C

[8] AICON 3D SYSTEMS GMBH, OPTOCAT 2015R2 - New version, new functions, 2015. [Online]. Available: https://bit.ly/3cvv8l7

[9] Stratasys, Manual del usuario uPrint SE and uPrint SE plus personal 3D printers, 2011. [Online]. Available: https://bit.ly/2T1H2eQ
[10] S. Belcher, Practical Extrusion Blow Molding, 1999. [Online]. Available: https://bit.ly/2WuzVha

[11] I. Suchy, Handbook of die design. McGrawHill, 2005. [Online]. Available: https://bit.ly/ 35 YaWGb

[12] L. Espinoza and C. Nuila, I18RE-MD001 Mediciones dimensionales a botellas de muestra. Laboratorio de Metrología Industrial, Soyapango, 2018.

[13] J. Hernández, Nota técnica: Principio de funcionamiento del sistema de inyección y análisis para la comprensión de la influencia del defecto rebaba de preformas PET. Universidad de Carabobo, Valencia, Venezuela, 2013.

[14] 3D Printers. (2018) Impresoras 3d para plástico. [Online]. Available: https://bit.ly/2Z2nISf 